\title{
The horizontal branch morphology of M 31 globular clusters ${ }^{\star}$ Extreme second parameter effect in outer halo clusters
}

\author{
S. Perina ${ }^{1,2}$, M. Bellazzini ${ }^{1}$, A. Buzzoni ${ }^{1}$, C. Cacciari $^{1}$, L. Federici ${ }^{1}$, F. Fusi Pecci ${ }^{1}$, and S. Galleti ${ }^{1}$ \\ 1 INAF - Osservatorio Astronomico di Bologna, via Ranzani 1, 40127 Bologna, Italy \\ e-mail: sibilla.perina2@unibo.it \\ 2 Departamento de Astronomía y Astrofísica, Pontificia Universidad Católica de Chile, 7820436 Macul, Santiago, Chile
}

Received 17 July 2012 / Accepted 4 September 2012

\begin{abstract}
We use deep, high quality color magnitude diagrams obtained with the Hubble Space Telescope to compute a simplified version of the Mironov index (SMI; $\frac{B}{B+R}$ ) to parametrize the horizontal branch (HB) morphology for 23 globular clusters in the M 31 galaxy (Sample A), all located in the outer halo at projected distances between $10 \mathrm{kpc}$ and $100 \mathrm{kpc}$. This allows us to compare them with their Galactic counterparts, for which we estimated the SMI exactly in the same way, in the SMI vs. $[\mathrm{Fe} / \mathrm{H}]$ plane. We find that the majority of the considered M 31 clusters lie in a significantly different locus, in this plane, with respect to Galactic clusters lying at any distance from the center of the Milky Way. In particular they have redder HB morphologies at a given metallicity, or, in other words, clusters with the same SMI value are $\approx 0.4$ dex more metal rich in the Milky Way than in M 31 . We discuss the possible origin of this difference and we conclude that the most likely explanation is that many globular clusters in the outer halo of M 31 formed $\approx 1-2 \mathrm{Gyr}$ later than their counterparts in the outer halo of the Milky Way, while differences in the cluster-to-cluster distribution of He abundance of individual stars may also play a role. The analysis of another sample of 25 bright M 31 clusters (eighteen of them with $M_{V} \leq-9.0$, Sample B), whose SMI estimates are much more uncertain as they are computed on shallow color magnitude diagrams, suggests that extended blue HB tails can be relatively frequent among the most massive M 31 globular clusters, possibly hinting at the presence of multiple populations.
\end{abstract}

Key words. stars: horizontal-branch - galaxies: star clusters: general - globular clusters: general - ultraviolet: stars galaxies: stellar content

\section{Introduction}

Globular clusters (GCs) are well studied and widely used tracers of the earliest epoch of galaxy formation. They are found in any kind of galaxy, they are bright and (typically) several Gyr old, at least in the local Universe. Probably the most useful property of GCs is that valuable estimates of their fundamental physical characteristics, notably metallicity and age, can be obtained with various techniques (see Brodie \& Strader 2006, for a recent review and references). Integrated colors and spectra can be used to study unresolved clusters in distant galaxies. In the range of distances in which GCs can be resolved into individual stars, color magnitude diagrams (CMD) can be derived and the most reliable and precise estimates of the cluster parameters can be obtained from observables defined on the CMD.

This is especially true for cluster age, as the luminosity of the main sequence turn off (MSTO) point is unrivaled as an age indicator (Renzini \& Fusi Pecci 1988; Gallart et al. 2005). The advent of large telescopes and modern CCD cameras brought the MSTO of all Galactic GCs within reach in the last two decades, thus allowing the establishment of a robust and accurate age scale (see Dotter et al. 2010, for a state-of-the-art analysis and references). Before this epoch, the key age indicator which was

* Based on observations made with the NASA/ESA Hubble Space Telescope, obtained from the Hubble Legacy Archive, which is a collaboration between the Space Telescope Science Institute (STScI/NASA), the Space Telescope European Coordinating Facility (ST-ECF/ESA) and the Canadian Astronomy Data Centre (CADC/NRC/CSA). STScI is operated by the Association of Universities for Research in Astronomy, Inc., under NASA contract NAS 5-26555. adopted to obtain global constraints on the Galactic halo formation from GCs was the horizontal branch (HB, Searle \& Zinn 1978; Zinn 1980). Low-mass stars $\left(m \lesssim 1 M_{\odot}\right)$ populate the HB in the evolutionary phase of core He burning. The actual temperature and luminosity of a given HB star at the beginning of this phase (Zero Age horizontal branch, ZAHB) is determined by the complex interaction of several different factors (Rood 1973). The overall metallicity is generally recognized as the most important parameter, but age, $\mathrm{He}$ abundance, stellar rotation, and any parameter affecting the mass loss along the red giant branch (RGB), may have a substantial role (Fusi Pecci et al. 1993; Catelan 2009). The realization that metallicity alone was not sufficient to account for the complex behavior of HB morphologies in Galactic GCs led to the so called second parameter problem, that dominated the scientific debate in the field of Galactic astronomy for a couple of decades (see, e.g., Sandage \& Wildey 1967; Zinn 1980; Preston et al. 1991; Fusi Pecci et al. 1993; Lee et al. 1994; Catelan \& de Freitas Pacheco 1994, 1995; Fusi Pecci \& Bellazzini 1997; Recío-Blanco et al. 2006; Dotter et al. 2010; Gratton et al. 2010, and references therein). The most recent and thorough analyses concluded that no less than three parameters are needed to account for the HB morphology of Galactic GCs (an idea already proposed in the past, see Fusi Pecci et al. 1993; Recío-Blanco et al. 2006), namely metallicity, age, and He abundance (Gratton et al. 2010).

The role of He abundance should be considered within the framework of the co-existence of multiple populations in globulars (see Gratton et al. 2012, for a recent review and references). In the most generally accepted view of the early evolution of GCs, subsequent generations of stars have undergone 
processing of the the light elements, indicative of hot H-burning via the CNO cycle, which includes the enrichment of He (see, e.g., Carretta et al. 2010, for discussion and references). This should lead to strong effects on the HB morphology of presentday clusters (see, e.g. D'Antona et al. 2005; Caloi \& D'Antona 2007; Yoon et al. 2008; Gratton et al. 2010).

While the use of the HB morphology as a possible (albeit ambiguos, Fusi Pecci \& Bellazzini 1997) age indicator has been superseded by much more reliable techniques for nearby clusters, it may still be valuable for more distant systems, where the MSTO of GCs is too faint to be observed with the instrumentation currently available. If we consider the case of our nearest neighbour giant galaxy M 31, the available observational material on GCs is quite similar to what was available for Galactic GCs in the seventies/early eighties, which led to the very influential scenario proposed by Searle \& Zinn (1978), i.e.:

- CMDs reaching down to a few magnitudes below the HB level are available for several GCs, thanks to Hubble Space Telecope (HST) observations (see, e.g. Ajhar et al. 1996; Fusi Pecci et al. 1996; Holland et al. 1997; Rich et al. 2005; Mackey et al. 2007; Perina et al. 2011, and references therein). It is possible to reach the MSTO luminosity of M 31 clusters, but this requires such a large amount of HST time as to make a systematic study unpractical (Brown et al. 2004).

- Global metallicity estimates from integrated spectra and/or from the color of the RGB are also available (Galleti et al. 2009; Caldwell et al. 2011; Perina et al. 2009), analogously to the MW GC compilation by Zinn \& West (1984).

In Federici et al. (2012, Paper I hereafter) we collected a dataset of $48 \mathrm{M} 31$ GCs having $V, V-I$ or $V, B-V$ CMD homogeneously derived by us from images collected by different groups ${ }^{1}$. In that paper, we used these CMDs to obtain an empirical calibration of the relation between the absolute $V$ magnitude of the HB $\left(M_{V}(\mathrm{HB})\right)$ and the metallicity $([\mathrm{Fe} / \mathrm{H}])$, as well as a new estimate of the distance to M 31. Here we use the same dataset, and the cluster parameters homogeneously obtained in Paper I, to get a first systematic outlook on the behavior of the HB morphology in the GC system of that galaxy, in particular in comparison with the Milky Way (MW), from the distribution of individual stars in each cluster (see Rey et al. 2007; Dalessandro et al. 2012, for an independent analysis based on integrated ultraviolet colors). We adopt a more straightforward parametrization of the HB morphology through a "simplified" version of the Mironov (1972) index MI $=\frac{B}{B+R}$. In the following we refer to this newly defined index as the simplified Mironov index (SMI; see Sect. 2.1 for details).

A previous use of SMI on a smaller sample of M 31 GCs was made by Rich et al. (2005), whereas a more basic approach to the HB morphology classification was successfully attempted by Perina et al. (2011), specifically aimed at verifying the effect of the $\mathrm{HB}$ morphology on the $\mathrm{H}_{\beta}$ spectral index.

The present analysis should be considered as a first attempt to provide a view of the M 31 GC system at a comparable level of accuracy as we had about 30 years ago for our own Galaxy (but with different selection biases, see Sect. 2.3), which is a remarkable step forward in the understanding of the formation and early evolution of M 31. In Sect. 2 we present our sample and assumptions, and we describe the procedure to obtain the HB morphology indicator used in the analysis. In Sect. 3 we compare

\footnotetext{
1 The sample includes also four objects classified as Extended Clusters (EC), since they appear old and metal-poor as classical globulars (see Mackey et al. 2006, and references therein).
}

the HB morphology of the considered M 31 clusters with that of MW GCs, and discuss the possible origins of the observed differences. Finally, in Sect. 4, we summarize and discuss our results also in the context of the literature.

\section{Data and data analysis}

From the dataset presented in Paper I we selected two samples of clusters for our analysis, depending on the quality of the available CMD:

- Sample A: 23 clusters with well defined CMD sequences and easily identifiable $\mathrm{HB}$, limiting magnitude $V_{0} \gtrsim 27.0$ (i.e. reaching approximately $2 \mathrm{mag}$ below the HB level, at least), and low contamination from the M 31 field. This constitutes the bulk of our analysis. The CMD for all the clusters in Sample A are shown in Fig. 1, to give the reader a clear idea of the quality of the HB morphology classification that can be obtained from this material. It must be noted that the quality of the data for Sample A clusters is not fully homogeneous. The CMDs of B384 and B468 have a brighter limiting magnitude than most of the other clusters of this sample. B008, B292, B298, B336, B337, B350, and B531 suffer from a slightly higher degree of contamination and/or crowding, w.r.t. to the other Sample A clusters, and/or may be affected by some differential reddening (still not seriously affecting their HB morphology).

- Sample B: 25 clusters with CMD not fulfilling the above criteria, still valuable, however, to obtain an estimate of SMI, albeit significantly more uncertain than for Sample A. We briefly consider Sample B here only for completeness and to get preliminary insight for future investigations.

In Paper I all the cluster CMDs were compared with a grid of RGB and HB templates of Galactic GCs with a fitting procedure that provides simultaneous best estimates of the $\mathrm{HB}$ magnitude level $V_{\mathrm{HB}}$, the reddening $E(B-V)$, the metallicity $[\mathrm{Fe} / \mathrm{H}]$, and the distance modulus for each cluster (see Paper I for a detailed description and discussion about uncertainties; we discuss the role of these uncertainties in our analysis in Sect. 3). In the following we adopt $V_{\mathrm{HB}}, E(B-V)$ and $[\mathrm{Fe} / \mathrm{H}]$ from Paper I; it is clear that Sample A clusters, having the best CMDs, have also the most reliable and accurate estimates of these parameters.

All the results of the present study are based on the comparison with Galactic GCs taken as a reference. As an homogeneous Galactic sample, we adopt the publicly available HST photometry of 74 Galactic GCs from Piotto et al. $(2002)^{2}$. The values of $[\mathrm{Fe} / \mathrm{H}], V_{\mathrm{HB}}, E(B-V)$ and $(m-M)_{0}$ for these clusters have been taken from Harris (1996, 2010 version). The adopted extinction laws are the same as in Paper I, $A_{V}=3.1 E(B-V)$, $A_{I}=1.94 E(B-V)$ and $E(V-I)=1.375 E(B-V)$. The list of Sample A and Sample B clusters and the relevant parameters for the present analysis are provided in Table 1.

\subsection{The simplified Mironov index (SMI)}

In the widely used original Mironov index $\mathrm{MI}=\frac{B}{B+R}$ (see e.g. Searle \& Zinn 1978; Lee et al. 1994; Catelan \& de Freitas Pacheco 1994, and references therein), $R$ and $B$ are the numbers

2 The original data (Piotto et al. 2002) were obtained in the F439W and $F 555 W$ bands of the Wide Field and Planetary Camera 2 (WFPC2). Photometry in these filters has been converted into standard $B, V$ photometry as described in Paper I. 

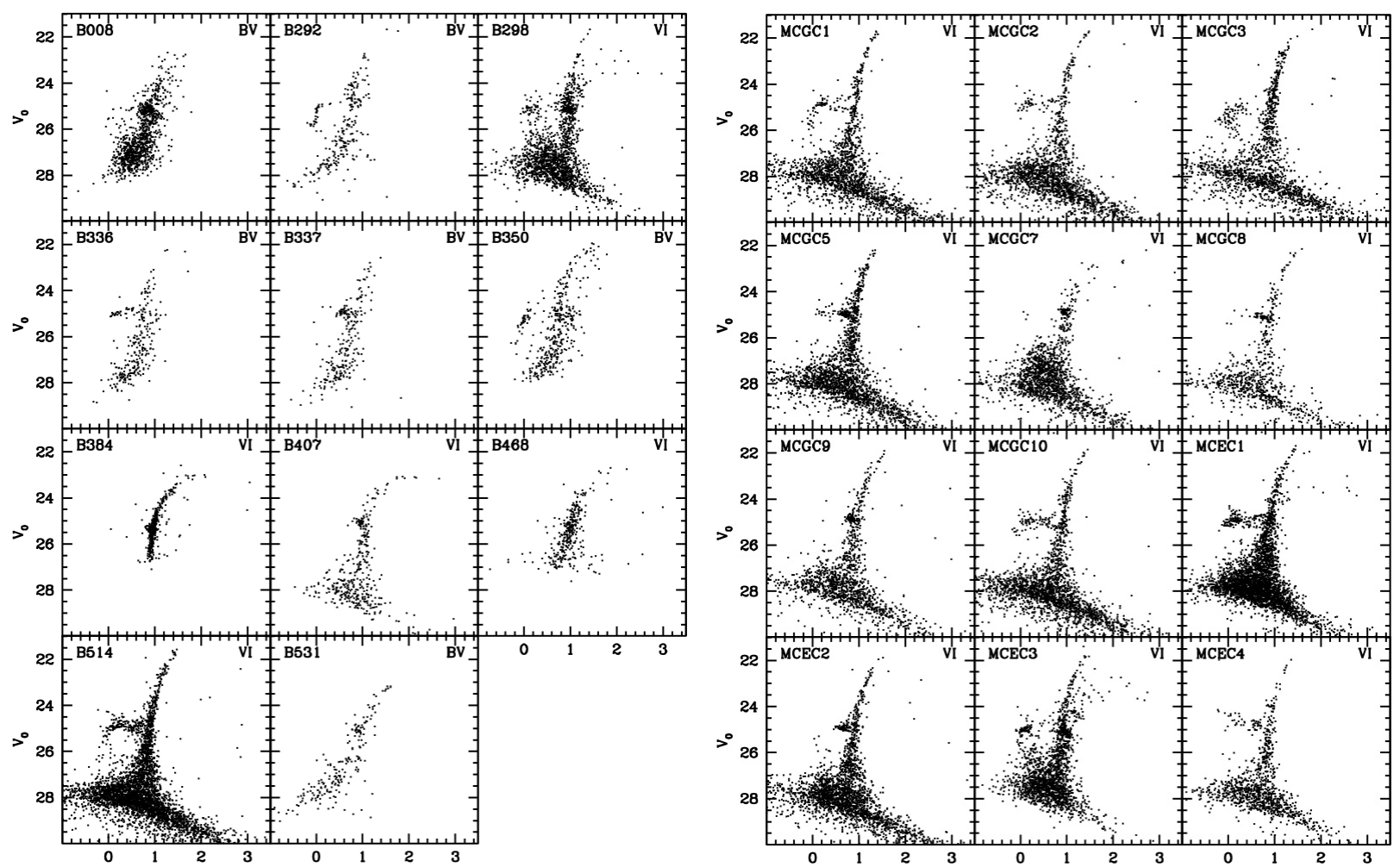

Fig. 1. Color-magnitude diagrams of the target clusters. The color on the $x$ axis is indicated by a label in the top-right corner of each panel: $\mathrm{BV}$ corresponds to the dereddened color $(B-V)_{0}$ and VI corresponds to the dereddened color $(V-I)_{0}$.

of HB stars respectively redder and bluer than the RR Lyrae instability strip edges. Here we use the same formulation, but $R$ and $B$ are defined as the number of HB stars redder and bluer than a given color threshold, approximately located in the middle of the RR Lyrae instability strip. Given the sometimes scanty population on the $\mathrm{HB}$, this appeared as the most sensible choice allowing us to account for all the HB stars with a single parameter.

We estimated the SMI from extinction-corrected CMDs as illustrated in Fig. 2 and described below:

1. For each cluster we consider a circular area around the center that is clearly dominated by cluster members. All the star counts described below are performed in this selected circle.

2. We count as $R$ the HB stars lying within \pm 0.5 mag from $V_{H B}$ and having $0.50<(V-I)_{0} \leq 0.80$ or $0.30<(B-V)_{0} \leq 0.65$, depending on the photometric bands of the available CMD.

3. We count as $B$ the HB stars having $\left(V_{\mathrm{HB}}-1.0\right)<V_{0}<26.0$, and $(V-I)_{0} \leq 0.50$ or $(B-V)_{0} \leq 0.3$, depending on the available CMDs. The $V_{0}<26.0$ limit has been adopted to avoid regions of the CMD were the completeness level can be sharply falling, thus strongly biasing the star counts. Note that the same selection boxes are adopted for M 31 and MW $\mathrm{GCs}^{3}$. This implies that a fraction of genuine and clearly identifiable Blue HB (BHB) stars can be excluded in some Galactic GCs (as in the case of NGC 6229, shown in Fig. 2), to preserve the full homogeneity in the comparison with M 31 clusters. The case of NGC 6229 shows also that the same threshold helps to prevent contamination of the

3 The $V_{0}<26.0$ limit for the selection of B stars adopted for M 31 clusters correspond to $M_{V}=1.58$, adopting the average distance modulus from Paper I, $(m-M)_{0}=24.42$. This has been converted into the corresponding limit for each Galactic GC adding their true distance moduli.

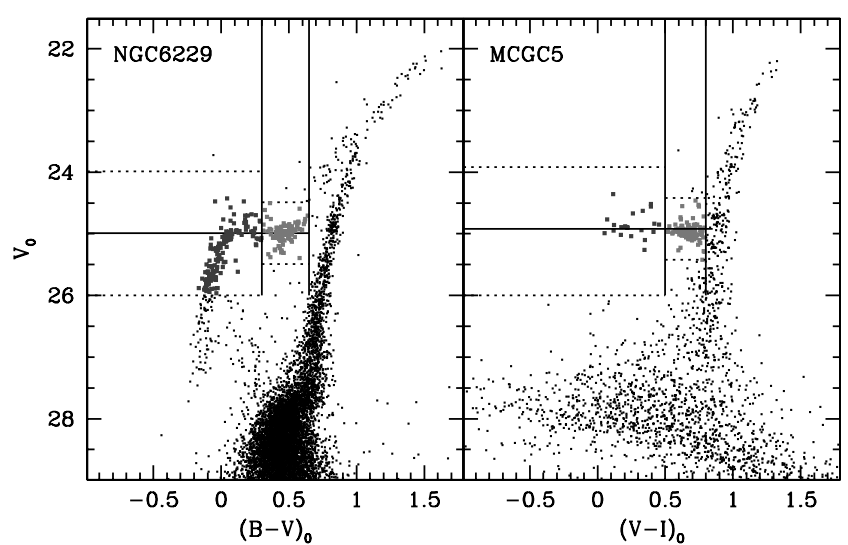

Fig. 2. Illustration of the selection boxes adopted for the computation of the SMI. Left panel: CMD of the Galactic globular cluster NGC6229, reported to the mean distance of M 31. Right panel: CMD of the Sample A globular cluster MCGC5. In each panel, the vertical segments mark, from left to right, the boundary between the blue (B) and red (R) part of the HB and the red limit of the HB selection. The continuous horizontal line is the mean level of the $\mathrm{HB}$, while the dotted horizontal segments enclose the stars selected as bona-fide HB. In both diagrams Blue and Red HB stars selected to compute SMI are plotted in dark and light grey, respectively.

BHB star counts from blue stragglers. In any case, stars appearing to be associated with the Blue Straggler sequence are excluded from the computation of SMI.

4. For Sample A clusters, the average number of field stars falling in each selection box was estimated using an annulus (field annulus) surrounding the cluster with the same area as the selected circle. Field counts are subtracted from the cluster counts and SMI is computed propagating Poisson errors 
Table 1. M 31 clusters parameters.

\begin{tabular}{|c|c|c|c|c|c|c|}
\hline Name & SMI & {$[\mathrm{Fe} / \mathrm{H}]$} & $E(B-V)$ & $\begin{array}{c}R_{\mathrm{p}} \\
{[\mathrm{kpc}]}\end{array}$ & $\begin{array}{c}R_{\mathrm{GC}} \\
{[\mathrm{kpc}]}\end{array}$ & Sample \\
\hline B008 & $0.00 \pm 0.10$ & -1.00 & 0.07 & 5.6 & 12.1 & A \\
\hline B292 & $0.91 \pm 0.25$ & -1.90 & 0.15 & 16.7 & 33.4 & A \\
\hline B298 & $\leq 0.76 \pm 0.22$ & -1.80 & 0.09 & 13.9 & 28.8 & A \\
\hline B336 & $0.55 \pm 0.26$ & -1.90 & 0.10 & 12.7 & 31.5 & A \\
\hline B337 & $0.00 \pm 0.10$ & -1.30 & 0.06 & 13.4 & 15.0 & A \\
\hline B350 & $0.83 \pm 0.31$ & -1.80 & 0.11 & 11.4 & 15.7 & A \\
\hline B407 & $0.00 \pm 0.10$ & -0.40 & 0.10 & 19.4 & 30.9 & A \\
\hline B514 & $0.61 \pm 0.12$ & -1.91 & 0.09 & 54.0 & 58.8 & A \\
\hline B531 & $0.00 \pm 0.10$ & -0.40 & 0.14 & 16.8 & 35.2 & A \\
\hline MCGC1 & $0.78 \pm 0.22$ & -2.15 & 0.12 & 45.3 & 46.9 & A \\
\hline MCGC2 & $0.77 \pm 0.26$ & -1.90 & 0.10 & 32.6 & 52.0 & A \\
\hline MCGC3 & $0.91 \pm 0.24$ & -1.90 & 0.10 & 31.1 & 39.1 & A \\
\hline MCGC5 & $0.16 \pm 0.05$ & -1.90 & 0.11 & 76.9 & 76.9 & A \\
\hline MCGC7 & $0.00 \pm 0.10$ & -0.70 & 0.06 & 17.7 & 75.7 & A \\
\hline MCGC8 & $0.14 \pm 0.08$ & -1.53 & 0.09 & 36.2 & 54.4 & A \\
\hline MCGC9 & $0.09 \pm 0.06$ & -1.40 & 0.16 & 38.0 & 68.2 & A \\
\hline MCGC10 & $0.66 \pm 0.18$ & -1.90 & 0.09 & 98.1 & 104.0 & A \\
\hline MCEC1 & $0.71 \pm 0.14$ & -1.91 & 0.10 & 13.0 & 25.2 & A \\
\hline MCEC2 & $0.11 \pm 0.05$ & -1.75 & 0.13 & 35.9 & 48.9 & A \\
\hline MCEC3 & $\leq 0.84 \pm 0.18$ & -1.91 & 0.09 & 13.7 & 13.7 & A \\
\hline MCEC4 & $0.47 \pm 0.18$ & -1.78 & 0.11 & 58.7 & 65.4 & A \\
\hline B384 & $0.00 \pm 0.10$ & -0.50 & 0.04 & 16.0 & 21.6 & A \\
\hline B468 & $0.00 \pm 0.10$ & -0.70 & 0.06 & 19.7 & 22.5 & A \\
\hline B006 & $0.00 \pm 0.10$ & -0.55 & 0.08 & 6.3 & 51.4 & B \\
\hline B010 & $0.82 \pm 0.26$ & -1.80 & 0.16 & 5.6 & 41.5 & B \\
\hline B012 & $0.94 \pm 0.29$ & -1.80 & 0.11 & 5.6 & 38.2 & B \\
\hline B023 & $0.00 \pm 0.10$ & -0.90 & 0.28 & 4.3 & 54.5 & B \\
\hline B027 & $0.26 \pm 0.15$ & -1.66 & 0.18 & 5.9 & 36.6 & B \\
\hline B045 & $0.39 \pm 0.26$ & -0.90 & 0.16 & 4.8 & 47.5 & B \\
\hline B058 & $0.50 \pm 0.33$ & -1.40 & 0.11 & 6.8 & 25.2 & B \\
\hline B088 & $0.80 \pm 0.21$ & -1.90 & 0.38 & 3.7 & 8.0 & B \\
\hline B158 & $0.61 \pm 0.39$ & -0.90 & 0.09 & 2.3 & 2.3 & B \\
\hline B220 & $0.39 \pm 0.20$ & -1.70 & 0.06 & 5.1 & 22.0 & B \\
\hline B224 & $0.43 \pm 0.19$ & -1.80 & 0.07 & 5.1 & 11.8 & B \\
\hline B225 & $0.00 \pm 0.10$ & -0.50 & 0.05 & 4.6 & 47.5 & B \\
\hline B233 & $0.51 \pm 0.32$ & -1.53 & 0.10 & 7.9 & 8.7 & B \\
\hline B240 & $0.63 \pm 0.37$ & -1.66 & 0.14 & 7.1 & 28.5 & B \\
\hline B293 & $0.93 \pm 0.21$ & -1.70 & 0.12 & 16.8 & 18.3 & B \\
\hline B311 & $0.98 \pm 0.33$ & -1.75 & 0.25 & 12.8 & 39.8 & B \\
\hline B338 & $0.68 \pm 0.20$ & -1.20 & 0.04 & 10.0 & 35.8 & B \\
\hline B343 & $0.86 \pm 0.26$ & -1.50 & 0.10 & 14.4 & 71.6 & B \\
\hline B358 & $0.84 \pm 0.16$ & -1.91 & 0.05 & 19.4 & 76.6 & B \\
\hline B366 & $0.48 \pm 0.11$ & -1.80 & 0.09 & 11.8 & 41.6 & B \\
\hline B379 & $0.00 \pm 0.10$ & -0.50 & 0.13 & 11.1 & 58.6 & B \\
\hline B386 & $0.32 \pm 0.19$ & -1.10 & 0.04 & 13.8 & 19.9 & B \\
\hline B405 & $0.88 \pm 0.20$ & -1.55 & 0.08 & 17.9 & 68.7 & B \\
\hline G001 & $0.43 \pm 0.09$ & -0.90 & 0.04 & 33.9 & 61.9 & B \\
\hline B255D & $0.00 \pm 0.10$ & -0.70 & 0.14 & 12.3 & 18.5 & B \\
\hline
\end{tabular}

Notes. The reported uncertainties on SMI are formal propagated Poisson errors on star counts. We arbitrarily assigned an uncertainty of 0.1 in SMI to clusters having only red HB stars. Note that SMI values can be (roughly) converted into HBR, for comparison with other samples, by inverting Eq. (1), i.e. $H B R=2 S M I-1.0$. Distance moduli, $[\mathrm{Fe} / \mathrm{H}]$, and $E(B-V)$ are taken from Paper $\mathrm{I}$. $R_{\mathrm{p}}$ is obtained adopting the mean distance modulus from Paper I for all the clusters, $(m-M)_{0}=24.42$. The three-dimensional distance from the center of the galaxy, $R_{\mathrm{GC}}$, is obtained adopting the individual distance moduli from Paper I and $(m-M)_{0}=24.42$ for the center of M 31 .

on the star counts. The effects of uncertainty in $V_{\mathrm{HB}}$ are negligible, because of the generous interval in $V$ that is considered in the selection of HB stars. The uncertainty in $E(B-V)$ can have a significant impact, in principle: we consider the case in detail in Sect. 3.

5. For Sample B clusters, we directly estimate $B$ and $R$ from statistically decontaminated CMDs (see Perina et al. 2009, and Paper I). This is a less robust procedure for star counts with respect to that adopted for Sample A, but it was the only viable one, since the identification of key CMD features was too uncertain without previous decontamination.

The location of the threshold between R and B stars is arbitrary and has been set only for convenience. However, since the aim is the comparison between two sets of GCs where the SMI has been computed in the same way, this cannot introduce any serious bias 
in the analysis. On the other hand, the consistency between the threshold in $(B-V)_{0}$ and $(V-I)_{0}$ has been checked for accuracy using clusters that have photometry in all the three passbands.

In a few clusters the red HB is totally or partially superposed to the RGB. In these cases we estimated the contribution of these stars to $R$ by constructing the luminosity function (LF) of RGB stars in the selected circle and in the corresponding field annulus. Once subtracted the latter from the former, to remove any possible contamination from field Red Clump stars, we look for residual peaks in the decontaminated LF that can correspond to the red HB. Great care is taken to discriminate between the red $\mathrm{HB}$ and the (fairly smaller and, generally, brighter) peak produced by the RGB bump. Then the number of stars in the red HB peak is estimated by subtracting the underlying RGB, interpolated in the region of the red HB peak. We checked several clusters in this way for the presence of red HB stars superposed to the RGB, and a significant signal was detected only for two clusters, MCGC8 (36 R stars) and MCEC1 (46 R stars). In some cases, like e.g. B468, although the red HB is clearly superposed to the RGB, the above procedure is pointless as SMI vanishes all the way. For B298 and MCEC3 the uncertainties on the number of R stars are somewhat larger because of a stronger field contamination. For these two clusters we provide only upper limits to their SMI, but the true value cannot be much different from the reported one.

\subsection{Relation with other HB morphology indicators}

It is clear that the SMI should have a similar behavior as the original MI which, in turn, is a simpler version of the more generally used horizontal branch Ratio $\mathrm{HBR}=\frac{B-R}{B+R+V}$ (where $V$ is the number of stars in the RR Lyrae instability strip, Lee et al. 1994). The two indices are tied by the relation (Preston et al. 1991):

$\frac{B}{B+R}=0.50+0.50 \frac{B-R}{B+R+V}$

Fig. 3a shows that the same relation provides an excellent fit also to the trend between SMI and HBR.

These indices trace essentially the peak (or the weighted mean) of the HB color distribution, this being especially true for the SMI because the $V_{0}<26.0$ limit makes it blind to stars in the extreme blue tail of the HB. Other parameters - e.g. $L_{\mathrm{t}}$ or $T_{\mathrm{eff}}^{\max }(\mathrm{HB})$ - are better suited to trace the extension of the HB, in particular of the blue tail, when present (Preston et al. 1991; Fusi Pecci et al. 1993; Recío-Blanco et al. 2006; Gratton et al. 2010).

It has been shown (Rey et al. 2007; Dalessandro et al. 2012) that the integrated near/far ultraviolet (NUV/FUV) - visible colors $(N U V-V)_{0}$ and $(F U V-V)_{0}$ are good tracers of the GC HB morphology. In principle they should be preferred to our SMI, because by definition they cannot be affected by the incompleteness problem that forced us to adopt the $V_{0}<26.0$ limit, and so are particularly sensitive (especially $\left.(F U V-V)_{0}\right)$ to the blue tails of the HB distributions (Dalessandro et al. 2012). However, these integrated colors are much more affected by reddening and contamination from hot non-HB stars (e.g. from the M 31 disk field population or the turn-off stars of the GC itself, especially $(N U V-V)_{0}$; see, e.g., Sect. 2.2.1). Therefore, SMI should provide a complementary view of the HB morphology, with respect to the UV-optical integrated colors.

In Fig. 3b we show that indeed SMI and $(N U V-V)_{0}$ broadly anti-correlate, and the behavior of Galactic and M 31 clusters

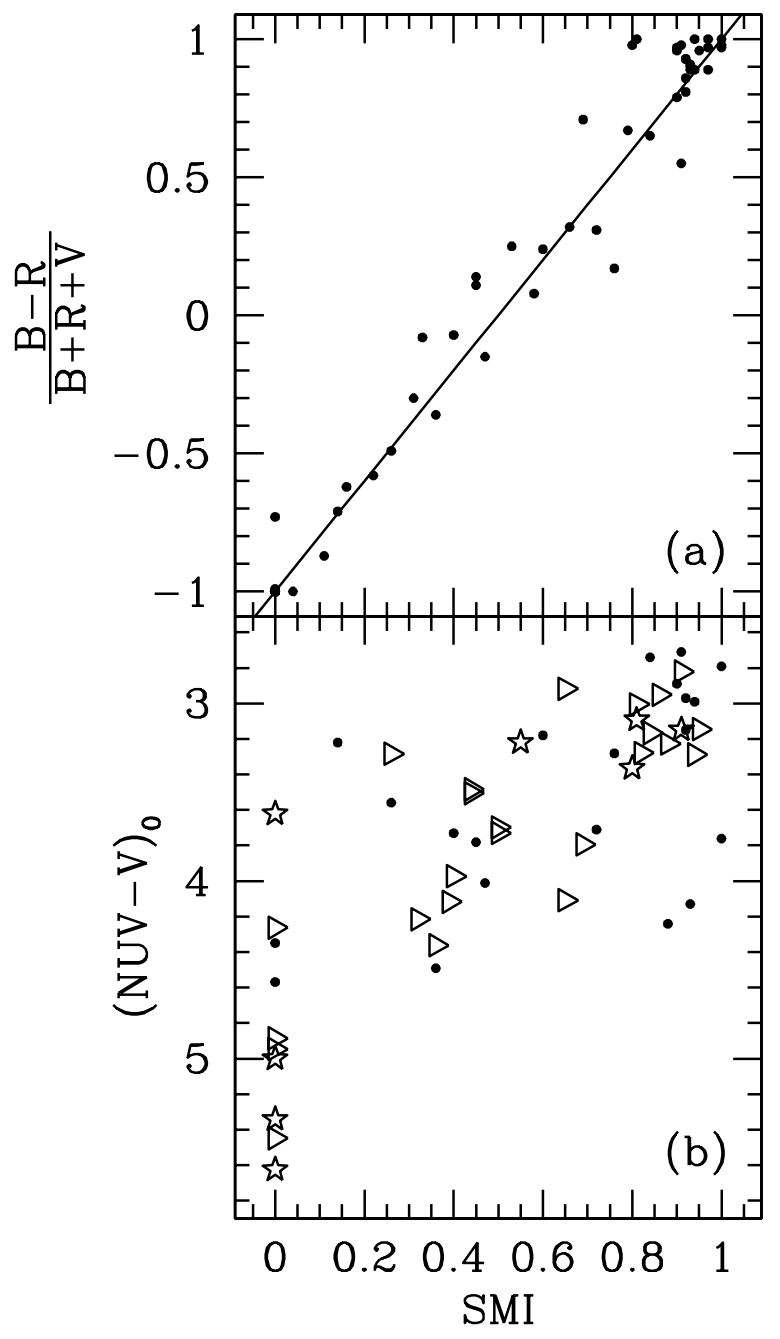

Fig. 3. Panel a): correlation of the SMI with the HBR index $\left(\frac{B-R}{B+R+V}\right)$ for our sample of Galactic GCs. HBR values are taken from the latest version of the Harris (1996) catalogue. The continuous line is the relation between $\frac{B}{B+R}$ and $\frac{B-R}{B+R+V}$ from Preston et al. (1991), reported in Eq. (1). Panel b): correlation of SMI with the integrated ultraviolet-optical color $(N U V-V)_{0}$ for Sample A (open stars), Sample B (open triangles, to be considered as lower limits), and Galactic GCs (small filled circles).

from our samples are very similar in this plane ${ }^{4}$. We note that most of the M 31 clusters in our samples having available NUV and FUV magnitudes are from Sample B. It is reassuring to note that, in spite of the large uncertainties associated to the SMI estimates for Sample B clusters, SMI anti-correlates quite well with $(N U V-V)_{0}$. The same degree of correlation is observed between $(N U V-V)_{0}$ and HBR (see e.g. Dalessandro et al. 2012).

Given the results illustrated in Fig. 3, we consider that our SMI is validated as a reliable HB morphology indicator tracing the peak of the HB distribution as MI and HBR. For most of the

\footnotetext{
${ }^{4}$ Integrated FUV and NUV magnitudes (from Galaxy Evolution Explorer - GALEX photometry) are taken from Rey et al. (2007), for M 31 clusters, and from Dalessandro et al. (2012) for MW clusters. The values of $(N U V-V)_{0}$ and $(F U V-V)_{0}$ have been computed adopting $A_{N U V}=8.90 E(B-V)$ and $A_{F U V}=8.16$ (from Rey et al. 2007), and taking $V$ magnitudes from RBC V4.0 and $E(B-V)$ from Paper I. The adoption of the very recent updated and extended dataset by Kang et al. (2012) would not lead to any significant improvement, for the sample of M 31 clusters considered here.
} 


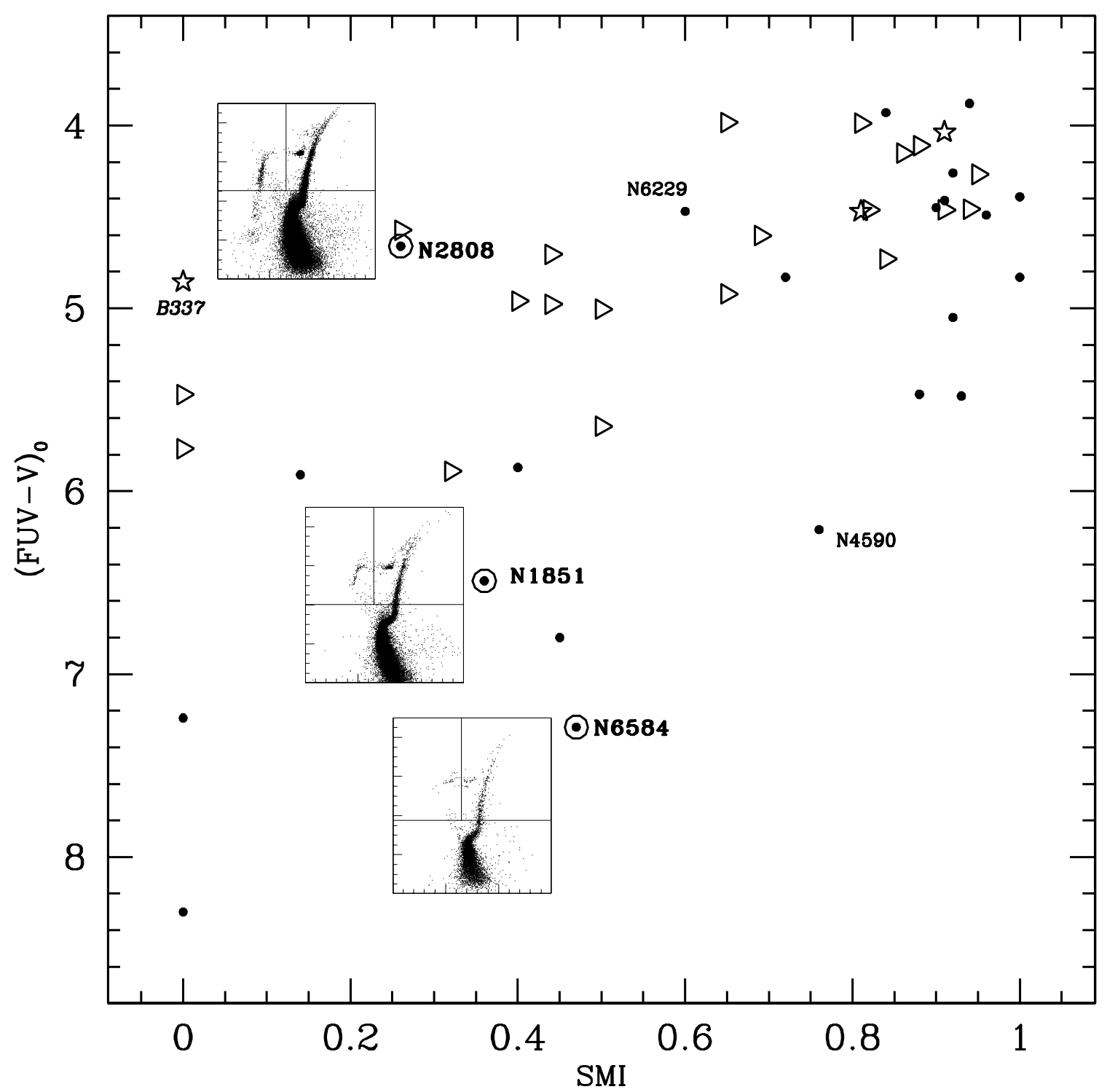

Fig. 4. The SMI versus the integrated ultraviolet-optical colors $(F U V-V)_{0}$ for Sample A (open stars), Sample B (open triangles, to be considered as lower limits) and Galactic GC (small filled circles). Three remarkable Galactic GCs are highlighted with a concentric open circle, are labelled in boldface and have their CMD presented in an inset box, to show the actual variation of their HB morphology with decreasing $(F U V-V)_{0}$ at nearly constant SMI. Two other Galactic clusters and one Sample A cluster, that are mentioned in the text, are also labelled.

clusters in Sample A which lack UV-optical colors, this is the only parametrization of the HB morphology currently available.

\subsubsection{SMI vs. $(F U V-V)_{0}$ : the effect of blue tails}

The comparison between SMI and $(F U V-V)_{0}$, shown in Fig. 4, deserves a deeper discussion. The anti-correlation is significantly less tight than in the SMI vs. $(N U V-V)_{0}$ plane and, above all, the distribution of M 31 clusters is different from their MW counterparts. In particular they are all confined in a relatively narrow strip along the blue edge of the $(F U V-V)_{0}$ distribution.

This is partially due to the selection in FUV flux that affects the M 31 sample. At that distance clusters with FUV fluxes below a certain threshold were not detected by GALEX: in practice M 31 GCs with $M_{V} \gtrsim-7.0$ do not have valid measures of the integrated FUV magnitude (Rey et al. 2007). This translates into a threshold in $(F U V-V)_{0}$ color ranging from $(F U V-V)_{0} \lesssim 7.2$ for the reddest clusters (having integrated $(B-V)_{0} \approx 1.0$ ) to $(F U V-V)_{0} \lesssim 5.0$ for the bluest ones (having integrated $\left.(B-V)_{0} \approx 0.3\right)$, effectively squeezing the M 31 GCs in our sample into a narrower range of $(F U V-V)_{0}$ with respect to MW clusters.

However, there are two additional effects that can concur to produce the observed difference. First, SMI estimates for Sample B clusters (the large majority of M 31 clusters in Fig. 4) should be considered as lower limits, because the bright limiting magnitude of their CMD may wipe out most (or all) of their BHB stars (if present) from the computation of SMI. Larger SMI values for some of these clusters would help to distribute them over the same range covered by Galactic GCs in this plane. Furthermore, some of them may have very extended blue tails, including a population of extreme HB (EHB) and/or (possibly) blue hook stars (see Catelan 2009, for references and discussion).

The inset CMD for three Galactic clusters (NGC 6584, NGC 1851 and NGC 2808) having similar SMI and widely different $(F U V-V)_{0}$ illustrates this possibility and shows very 
clearly the complementarity of SMI and $(F U V-V)_{0}$ in describing the HB morphology. All the three clusters have a bimodal HB morphology, however they greatly differ in the extension of their blue tail. The hottest temperature reached by their HB corresponds to the largest integrated FUV flux and, consequently, the bluest $(F U V-V)_{0}$ color (see Dalessandro et al. 2012). The difference in the morphology between NGC 4590 and NGC 6229 (also labelled in the figure and discussed in Sect. 3, below) is similar to that between NGC 6584 and NGC 1851/NGC 2808.

The trend shown in the CMDs indicates that a remarkably extended BHB is required to reach the bluest limits of the $(F U V-V)_{0}$ range, thus suggesting that several sample B clusters likely have a significant blue tail population that went undetected in their shallow CMDs. Hence extreme HB morphologies like that displayed by NGC 2808, and a few other peculiar clusters in the Milky Way, i.e. $\omega$ Cen, M 54 and NGC 2419, may be relatively common among Sample B clusters (see also Dalessandro et al. 2012). Interestingly enough, such extreme $(F U V-V)_{0}$ colors indicate that a substantial fraction (3-6\%, Buzzoni et al. 2012) of the total (bolometric) luminosity for these clusters is emitted shortward of $2500 \AA$, a feature that may closely deal, on a larger galactic scale, with the striking phenomenon of the UV upturn, as extensively observed among elliptical galaxies (Yi \& Yoon 2004).

The case of B337 is especially interesting since it has $\mathrm{SMI}=0.0$, i.e. purely red $\mathrm{HB}$ as seen from its CMD, and extremely blue $(F U V-V)_{0}$ color. Still, it is a sample A cluster and in Fig. 1 it can be appreciated that no significant BHB population emerges in the CMD down to $\approx 3$ mag below the red $\mathrm{HB}$ level, a range containing the majority of BHB stars in NGC 2808. A detailed inspection of the available HST images revealed the presence of two possible contaminating sources lying within the aperture adopted by Rey et al. (2007) for their NUV/FUV GALEX photometry $\left(r_{\text {ap }}=4.5^{\prime \prime}\right)$. A star nearly two magnitudes brighter than the brightest cluster star is located at $\approx 3.5^{\prime \prime}$ from the cluster center. Its color $\left((B-V)_{0}=0.45\right)$ and magnitude are typical of a foreground Galactic dwarf. While this bright star is bluer than most of the cluster giants and clearly contaminates the integrated FUV magnitude of the cluster, it is too red to push the integrated $(F U V-V)_{0}$ to the extreme value observed. Another potential contaminant has been identified at $\approx 0.25^{\prime \prime}$ from the cluster center, a region where photometry packages are unable to reliably resolve and estimate fluxes of any star. A very rough estimate based on the comparison of the intensity peak with stars of known magnitudes suggest that this star is brighter than the cluster RGB tip and may have a color as blue as $(B-V)_{0} \approx 0.0$. This would be consistent with a Post Asymptotic Giant Branch star (Jasniewicz \& Parthasarathy 2009), that may well dominate the UV flux of the cluster. Hence, B337 may be the example of a cluster whose integrated UV-optical color is strongly affected by fore/back-ground contamination and/or by a rare non-HB source. However, these cases should be quite unusual in our samples.

\subsection{An obvious selection bias}

Sample A is clearly not representative of the whole M 31 GC system. The quality criteria imposed on the CMD are very hard to fulfill for clusters that are projected on the high surface brightness bulge or disk of M 31, since there (a) the background level and the crowding are high, preventing accurate photometry of individual stars down to the required faint magnitude limit,

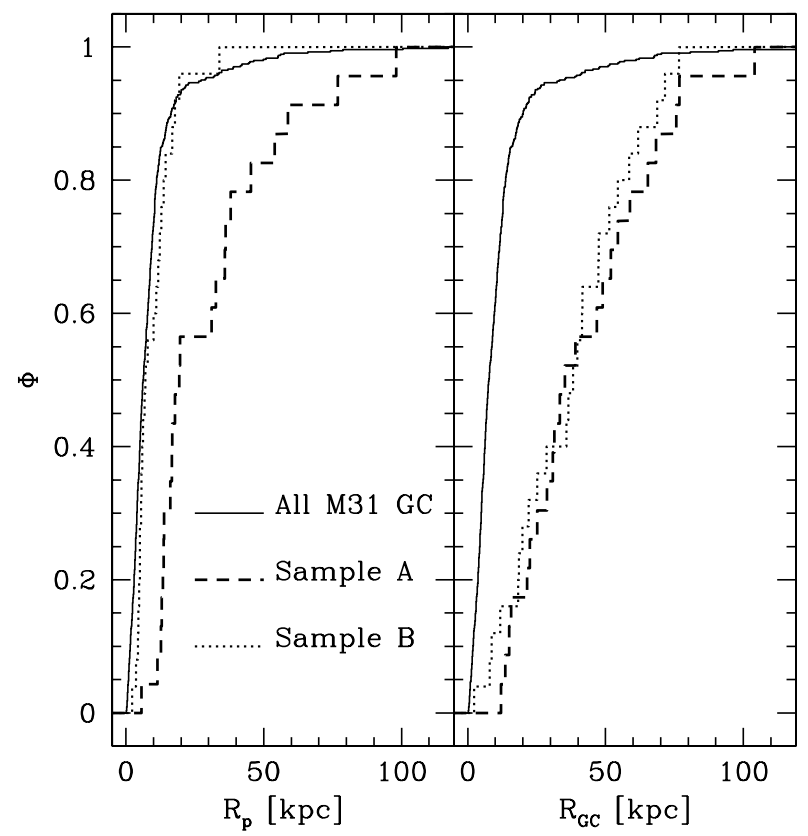

Fig. 5. Left panel: cumulative distributions of projected distances from the center of M 31 of Sample A (long dashed line) and Sample B (dotted line) are compared with the distribution of all the confirmed M 31 clusters classified as old (GCs) (solid line). Right panel: the same comparison but for the distributions of three-dimensional distances from the center of M $31\left(R_{\mathrm{GC}}\right)$. For the whole sample we used $\sqrt{\frac{3}{2} R_{\mathrm{p}}}$ as a proxy for $R_{\mathrm{GC}}$.

and (b) the contamination by field M 31 stars can be also very high, strongly affecting star counts on the HB. Moreover,clusters more distant from the center of their parent galaxy have intrinsically larger sizes (van den Bergh 1994), hence remote M 31 clusters are less severely crowded than those near the center, in average, when seen from the Earth.

Indeed, the left panel of Fig. 5 shows that most of Sample A clusters lies at projected distances $R_{\mathrm{p}}>10 \mathrm{kpc}$ from the center of $\mathrm{M} 31$, a radius that encloses approximately 80 per cent of the whole sample of M $31 \mathrm{GCs}^{5}$. Moreover, $\approx 20$ per cent of Sample A clusters have $R_{\mathrm{p}}>40 \mathrm{kpc}$, a range that contains less than 5 per cent of the whole sample. Finally, many of the outermost clusters of Sample A have been found to be likely associated with known existing substructures in the outer halo of M 31 (Perina et al. 2009; Mackey et al. 2010). Therefore, Sample A is more likely representative of the outer halo population of the GC system of M 31, and/or of the population of GCs more recently accreted via the disruption of their parent dwarf galaxies. This selection bias should be kept in mind very clearly when comparing Sample A with Galactic globulars.

Sample B shows a much more concentrated distribution of $R_{\mathrm{p}}$, more similar to the overall population. However, the right panel of Fig. 5 shows that the distribution of spatial (3D) distances ${ }^{6}$ is very similar to the one of Sample A, and clearly also not representative of the overall M 31 GC system.

5 The data are from the Revised Bologna Catalogue of M31 globular clusters and candidates Galleti et al. (2004, RBC V4.0); http: /www . bo.astro.it/M31/

${ }^{6}$ Derived by combining the projected distances and the line-of-sight distances from the center of M 31, the latter obtained from the individual distance moduli from Paper I, as done also by Mackey et al. (2006, 2007). 


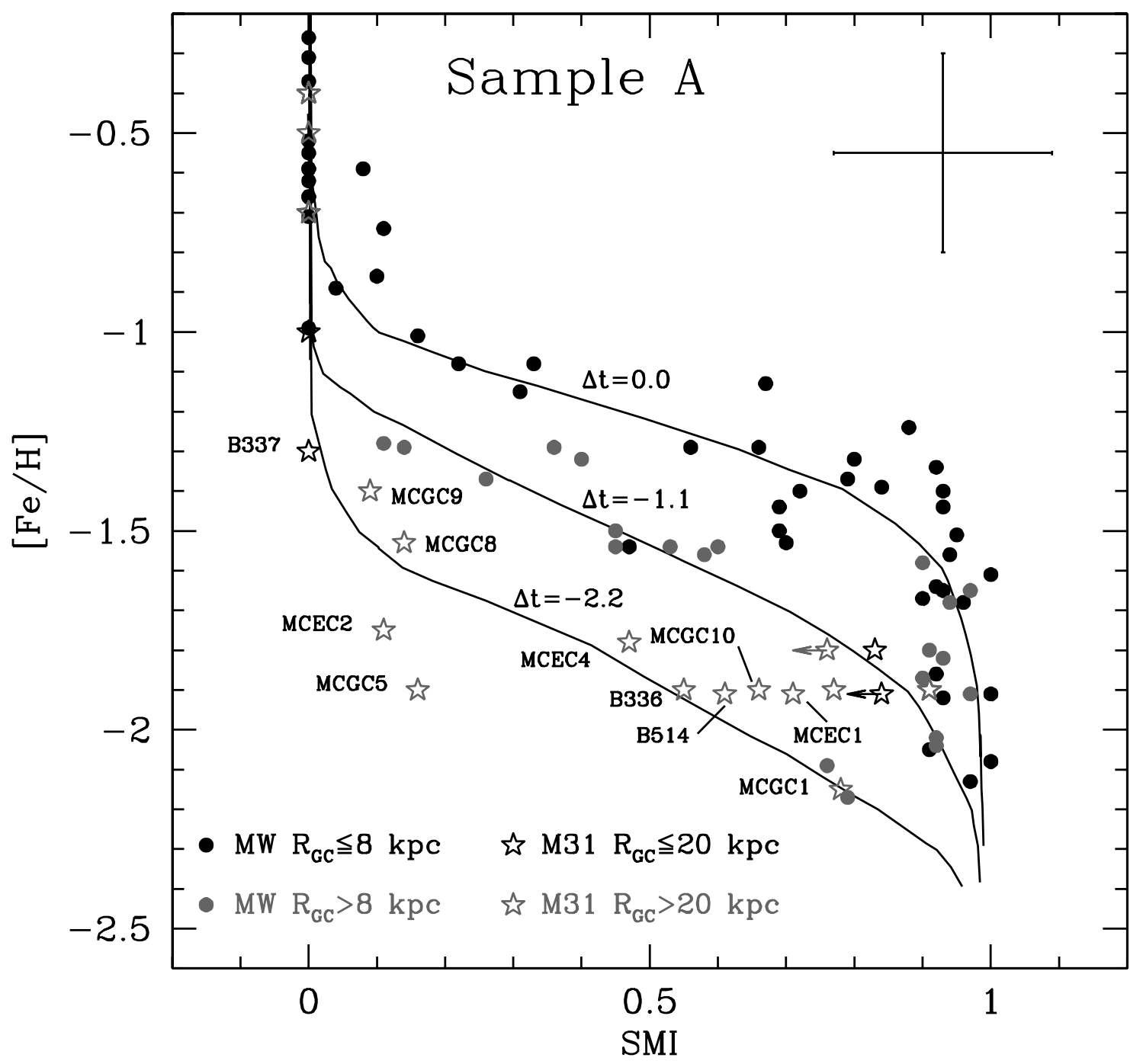

Fig. 6. The SMI vs. metallicity diagram. The Galactic GCs are plotted as filled circles, the M 31 Sample A GCs as open stars. The symbol color code shows the galacto-centric distance of the cluster, as described in the bottom-left corner of the panel. The $R_{\mathrm{GC}}=20 \mathrm{kpc}$ threshold was somehow arbitrarily adopted for Sample A clusters to highlight those that cannot be considered as exceptionally far, since it correspond to the radius containing $\approx 90 \%$ of the whole population of confirmed M 31 GCs (see Fig. 5). Arrows indicate upper limits. Solid lines are isochrones from the synthetic HB models by Rey et al. (2001), and are labelled according to their age difference in Gyr. Typical error-bars for M 31 GCs are shown in the top-right corner. Sample A clusters lying near the $\Delta t=-2.2 \mathrm{Gyr}$ isochrone are labelled.

It is worth recalling that Mackey et al. (2006) and Mackey et al. (2007) noted a strong second parameter effect among the recently discovered group of remote (and/or extended) clusters of M 31 (Huxor et al. 2004, 2005; Galleti et al. 2006). However, a quantitative and systematic comparison with MW clusters is attempted here for the first time.

\section{Results}

The main result of the present analysis is shown in Fig. 6. Sample A clusters are compared with MW clusters in the classical plane opposing metallicity and HB morphology. The solid lines show three theoretical isochrones illustrating the expected trend of the SMI as a function of $[\mathrm{Fe} / \mathrm{H}]$. These have been obtained by converting isochrones in the $\frac{B-R}{B+R+V}$ vs. $[\mathrm{Fe} / \mathrm{H}]$ plane (derived by Rey et al. 2001, from synthetic HB populations models) using Eq. (1).

It must be stressed that these isochrones are plotted only as a general reference for the interpretation of the observed distributions, since they (a) refer to a slightly different quantity than that plotted in Fig. 6 (i.e. the original MI instead of SMI, but this does not seem a reason of serious concern, see Fig. 3), and (b) the details of their shape depend on the mass loss recipe adopted in modeling the synthetic HB (Rey et al. 2001; Lee et al. 1994; Catelan \& de Freitas Pacheco 1994). The three isochrones correspond to different ages in steps of $1.1 \mathrm{Gyr}$, setting a rough relative age scale, from top to bottom $\Delta t=0,-1.1$ and $-2.2 \mathrm{Gyr}$ (see Fig. 9 in Rey et al. 2001). Alternatively, they can be read as a relative scale of He abundance $Y$, approximately corresponding to $\Delta Y=+0.019,0.0$ and -0.019 (Catelan, priv. comm. ${ }^{7}$. We stress that, for the same reasons mentioned above for the age scale, this should be considered just as a rough indication of the effect of $Y$ on SMI. It is also important to keep in mind

7 The adopted standard $Y$ abundance is $Y=0.23+2 Z$, where $Z$ is the fractional abundance of heavy elements (Yi et al. 2001). See Fig. 9 of Rey et al. (2007) for an example of how the shape of this kind of HB-morphology isochrones can be changed by the assumption of a significant spread in He abundance within the HB population. In our case, the presence of a population of He-rich stars would prevent the isochrones from reaching SMI values near zero even at high metallicities. 
that in our current view of globular clusters, a previously usual concept like "the He abundance of a given cluster" is no more valid, since all GCs presenting a spread in light elements are expected to present a spread also in $Y$ (see Sect. 1). Hence each of them is characterized, in this respect, by the distribution of He abundances of its member stars. Different distributions of $Y$ would lead to differences in HB morphology and, consequently, in SMI. However both the average abundance and the size of the spread (as well as the shape of the distribution) would concur in determining the final SMI value of a given cluster. Hence any difference in SMI caused by helium, should trace a difference in the $Y$ distributions of the considered clusters.

Figure 6 shows the well known fact that clusters residing in the outer halo $\left(\mathrm{OH}, R_{\mathrm{GC}}>8.0 \mathrm{kpc}\right)$ of the $\mathrm{MW}$ tend to lie on an isochrone corresponding to a younger age with respect to clusters in the Inner Halo $\left(\mathrm{IH}, R_{\mathrm{GC}} \leq 8.0 \mathrm{kpc}\right.$ Lee et al. 1994; Catelan \& de Freitas Pacheco 1994, 1995; Rey et al. 2001). This is especially evident at intermediate metallicity, since at the extremes of the metallicity range the HB morphology parameters like SMI saturate (i.e. are not able to discriminate within HB distributions that have only red or only blue stars). The recent analyses by Gratton et al. (2010), Dotter et al. (2010), and, in particular, Dotter et al. (2011) confirm that the observed difference largely traces actual age differences between the two subgroups of Galactic GCs: while IH clusters are approximately coeval at any metallicity, several $\mathrm{OH}$ clusters with $[\mathrm{Fe} / \mathrm{H}] \gtrsim-1.5$ are $\approx 1-2$ Gyr younger than their IH counterparts with the same metallicity.

The completely new feature of Fig. 6 is the location of M 31 GCs: most Sample A clusters with $[\mathrm{Fe} / \mathrm{H}] \leq-1.2$ lie in a different locus with respect to both $\mathrm{IH}$ and $\mathrm{OH}$ Galactic GCs, approximately along the isochrone corresponding to $\Delta t=-2.2 \mathrm{Gyr}$. Specifically, the clusters lying near (or below) the $\Delta t=-2.2 \mathrm{Gyr}$ isochrone are: B337, MCGC9, MCGC8, MCEC2, MCGC5, MCEC4, B336, B514, MCGC10, MCEC1, MCGC1. Therefore, eleven out of seventeen Sample A clusters in the considered metallicity range, i.e. more than half the sample, lies near this locus. These GCs (all labelled in Fig. 6) appear to have significantly redder HB morphology with respect to their Galactic counterparts of the same metallicity. From a different perspective, one can say that a given SMI value is reached by these M 31 clusters at a lower metallicity by $\approx-0.4$ dex than their Galactic counterparts. In the following we will refer to these eleven M 31 clusters as Anomalously Red (HB) Sample A clusters, abbreviated with the acronym ARSA. Note also that, for $[\mathrm{Fe} / \mathrm{H}] \lessgtr-0.8$, no Sample A cluster lie near the $\Delta t=0.0$ isochrone, along which most of the Galactic GCs are clustered.

Only two Galactic GCs are lying on the $\Delta t=-2.2 \mathrm{Gyr}$ isochrone as the ARSA clusters, i.e. NGC 4590 and NGC 7078. Both clusters are very metal-poor $([\mathrm{Fe} / \mathrm{H}] \lesssim-2.0)$ and are not far from the blue saturation limit of the SMI. The inspection of the CMD of NGC 7078 reveals that the faint limit imposed by our definition of SMI $(V<26.0$ for M 31 clusters $)$ cuts out a large number of blue HB stars belonging to the extended blue tail, namely $\approx 30 \%$ of the whole HB population. Therefore, in this case the SMI does not provide a good parametrization of the HB morphology, which is actually bluer than indicated by the SMI. We note, however, that virtually all the blue HB stars excluded from the SMI estimate for NGC 7078 lie within $\approx 1.5 \mathrm{mag}$ of the threshold (similar to the case of NGC 6229, illustrated in Fig. 2). Figure 1 shows that none of the ARSA clusters seems to have such a large population of blue HB stars in this range of magnitude. So it is very unlikely that the SMI of these clusters can be severely affected by this kind of problem.
On the other hand, no HB star of NGC 4590 lies below the faint limit of the selection, and hence the SMI accounts for the whole $\mathrm{HB}$ distribution, and its relatively low value traces a real difference in HB morphology between this cluster and other GCs of similar metallicity. This peculiarity is remarked also by Dalessandro et al. (2012), who showed that there is a deficiency of very blue HB stars in this cluster with respect to, for example, NGC 7099. Since NGC 4590 appears to be as old as the other metal-poor GCs of the MW, Dalessandro et al. (2012) suggest that the observed difference in HB morphology may be accounted for by a difference in He abundance ${ }^{8}$. However, it has to be recalled that the apparent anomalous position of these clusters may also be due to inadequacies of the theoretical models, especially in this low metallicity regime, where the shape of the isochrones may strongly depend on the assumptions on massloss (see Zinn 1993; Catelan 2009, for examples and discussion).

Obviously, the unusual location of an individual M 31 GC in the SMI vs. $[\mathrm{Fe} / \mathrm{H}]$ plane may be considered as hardly significant given the large uncertainties. However, to appreciate the actual sensitivity of SMI for the best Sample A clusters it may be interesting to consider the cases of MCGC5 and B514. Both clusters have very deep and clean CMDs, they have the same metallicity and they differ by $0.45 \pm 0.13$ in SMI, formally $\mathrm{a} \approx 3.5 \sigma$ difference. The comparison of their CMDs (in Fig. 1) reveals at a first glance that the HB morphology of B514 is indeed much bluer than that of MCGC5, even if both clusters have stars to the red and to the blue of the SMI B-to-R threshold. The same visual comparison can be made for all the ARSA clusters: the cluster-to-cluster differences measured by SMI corresponds to real differences in HB morphology that, in most cases, can be appreciated simply by looking at the CMDs. Moreover, Sample A clusters clearly display a collective behavior that is different from MW GCs (and similar to dwarf galaxy satellites of the MW and M 31, see Yang \& Sarajedini 2012), thus strongly hinting at a real difference in some fundamental physical parameter between the samples.

\subsection{The effect of systematics}

Since the observed difference seems to involve the large majority of Sample A clusters, it would be easily explained by a systematic error in one of the relevant parameters, i.e. metallicity and reddening, or SMI itself.

\subsubsection{SMI}

The most obvious candidate for a systematic effect is our HB morphology parameter. If large numbers of BHB stars were missed in our computation, the actual SMI would be mere lower limits, as in the case of Sample B (see Sect. 3.2). Once taken into account, these hypothetical BHB stars would lead ARSA clusters to move near the MW clusters, at least those residing in the outer halo of the MW.

However, the inspection of the CMD of ARSA clusters (Fig. 1) reveals that there is no sign of such a significant additional $\mathrm{BHB}$ population down to $\approx 3$ mag below the $V_{\mathrm{HB}}$ level.

\footnotetext{
8 We note that the two couples of clusters considered by Dalessandro et al. (2012), i.e. NGC 4590 vs. NGC 7099 and NGC 5466 vs. NGC 6341, display also a large difference in $M_{V}$ and central density which may play a role in the chemical enrichment of cluster stars. The correlation between these parameters and the extension of blue tails has been noted and discussed in Fusi Pecci et al. (1993) and Recío-Blanco et al. (2006).
} 
Thus, any hidden BHB should be made by extreme HB and/or blue hook stars, like the faintest HB stars in NGC 2808, i.e. stars that would be excluded from the computation of SMI by definition and cannot be at the origin of the difference between M 31 and MW GCs shown in Fig. 6. Moreover, the presence of a significant population of such stars after a gap in the HB distribution of $\gtrsim 2$ mag would make the overall morphology of ARSA clusters exceedingly peculiar, calling for an interpretation as the results discussed here.

\subsubsection{Metallicity}

A systematic underestimate of the metallicity of M 31 clusters by $\approx 0.4$ dex would move all the ARSA clusters on the same isochrone populated by Galactic $\mathrm{OH}$ clusters. However, such a large systematic error can be safely excluded. First, the metallicity estimates used here have been obtained in Paper I from the comparison of the observed RGB with templates of Galactic GCs, and hence the adopted metallicity scale is exactly the same for the MW and the M 31 samples (except for a possible factor that is discussed below). Second, in Paper I we have compared our $[\mathrm{Fe} / \mathrm{H}]$ estimates with three sets of independent spectroscopic estimates and we found that systematic differences are at most $0.1-0.2 \mathrm{dex}$ and in any case consistent with zero (see Fig. 6 of Paper I). It is particularly reassuring that the agreement with estimates from FeI lines from high resolution spectroscopy by Colucci et al. (2009) is excellent $\left(\Delta[\mathrm{Fe} / \mathrm{H}]_{\mathrm{CMD}-\mathrm{FeI}}=-0.04 \pm 0.21\right)$.

An implicit assumption in the metallicity scale of Paper I is that M 31 clusters are as enhanced in $\alpha$-element abundance (w.r.t the Sun) as their Galactic counterparts. This assumption was found to be valid, at least for the handful of bright M 31 clusters analyzed by Colucci et al. (2009). However this is not necessarily true for Sample A clusters. If these clusters had a solar $[\alpha / \mathrm{Fe}]$ ratio, the comparison with the grid of templates of $\alpha$-enhanced Galactic GCs would lead to underestimate the actual metallicity. Using the formulae provided by Ferraro et al. (1999) we estimated that in this case the $[\mathrm{Fe} / \mathrm{H}]$ values of Sample A clusters should be increased by +0.2 dex to keep them in the same metallicity scale as the Galactic GCs. This would reduce the difference between the M 31 and the MW clusters shown in Fig. 6 but it would not be sufficient to cancel it out, since the observed effect is 2 times larger. Even if a difference in $[\alpha / \mathrm{Fe}]$ were responsible for part of the observed effect, this would be nevertheless an important difference in a fundamental physical parameter of the clusters (i.e. the $[\alpha / \mathrm{Fe}]$ ratio), revealed thanks to Fig. 6.

\subsubsection{Reddening}

The other parameter that can produce a systematic effect is reddening. Like metallicity and distance, reddening was estimated simultaneously in Paper I by a best-fit of the CMD with the grid of MW GC templates, and it has been verified that the best-fit value cannot be changed by more than \pm 0.02 mag without compromising the overall fit by obtaining unrealistic (or nonsense) values of distance and metallicity. Moreover, changing the reddening has little direct effect on the final SMI value (by shifting the Blue/Red threshold), while it may have a sizable effect on the estimated metallicity. It turns out that to obtain the higher $[\mathrm{Fe} / \mathrm{H}]$, which would be necessary to reduce the difference between M 31 and MW clusters in Fig. 6, a lower reddening must

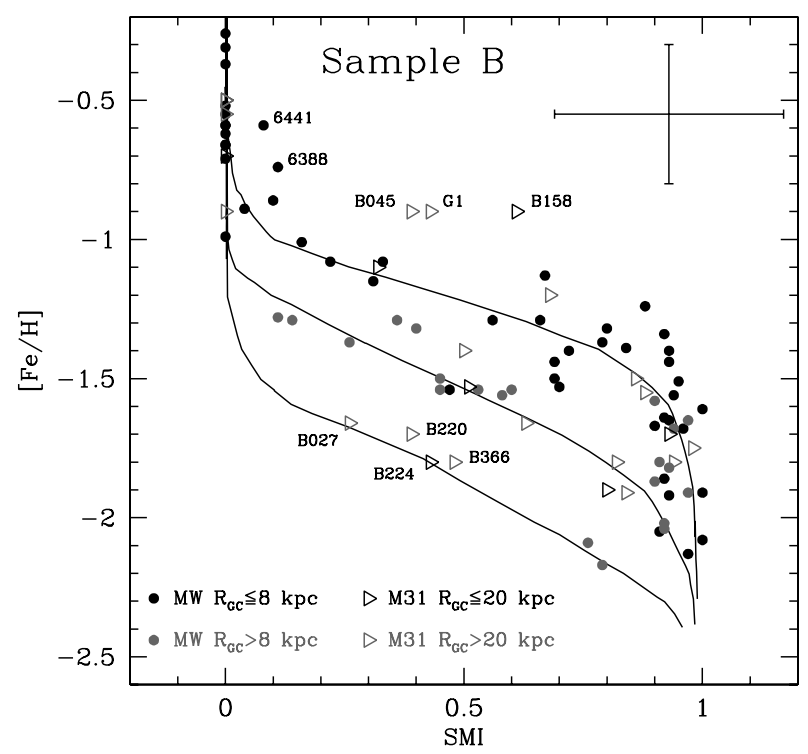

Fig. 7. Same as Fig. 6 but for Sample B M 31 clusters. All the estimates should be considered as lower limits, since the bright limiting magnitude of the available CMD does not allow to reveal a Blue Tail HB component, if present. The metal-rich Galactic GCs displaying bimodal HB morphology NGC 6441 and NGC 6388 are indicated with labels. Sample B clusters discussed in the text are also labelled.

be adopted with respect to Paper I. However, the comparison of our reddening values with three independent sets of estimates demonstrates that, if any small systematic effect were there, it would go in the opposite sense, since our $E(B-V)$ are on average a few hundredths of a magnitude smaller than those estimated by other studies (see Paper I for details).

However, for the sake of completeness, we have repeated, for the ARSA clusters, the whole procedure of metallicity estimate performed in Paper I and the computation of SMI, but forcing lower reddening values by 0.02 mag with respect to the bestfit solutions of Paper I. It turns out that (a) the changes in SMI are negligible $(\leq 0.05)$, and $(b)[\mathrm{Fe} / \mathrm{H}]$ values typically decrease by 0.1 dex, with a range between 0.05 dex and 0.25 dex, in agreement with the predictions by Buzzoni (1995, his Eq. (19)). While this clearly helps in reducing the difference between Sample A and the MW sample, it is not sufficient to close the gap, and for this "partial result" we pay a toll: all the CMD fits obtained with the lower $E(B-V)$ values are significantly worse than what found in Paper I, and in most cases clearly not acceptable.

In conclusion, it seems very unlikely that a systematic misestimate of $[\mathrm{Fe} / \mathrm{H}]$ or $E(B-V)$ can be the (only) cause of the different behavior of Sample A and MW clusters reported in Fig. 6.

\subsection{Sample B}

In Fig. 7 we show the distribution of Sample B clusters in the SMI vs. $[\mathrm{Fe} / \mathrm{H}]$ plane. Unfortunately, the uncertainties are too large to draw any firm conclusion, as most of the clusters in the interesting range of metallicity have just rough estimates of their SMI. In particular, all the SMI estimates for Sample B clusters should be considered as lower limits, since the bright limiting magnitude of the available CMDs does not allow to reveal even the reddest portion of the BHB component, if present. However, the comparison with other independent tracers of the HB morphology shown in Fig. 4 demonstrates that the estimated SMI may still carry useful information on the HB of these clusters. 
Taking Fig. 7 at face value, it can be noted that several Sample B clusters lie on the isochrones that are populated also by MW GCs, even at intermediate metallicity. Still, there are a few potentially interesting outliers:

- four anomalous clusters lying on the $\Delta t=-2.2 \mathrm{Gyr}$ isochrone, as most Sample A clusters do, namely B027, B220, B224, and B366 (the last has been highlighted and discussed also by Dalessandro et al. 2012, because of its anomalously red $(F U V-V)_{0}$ color);

- three metal-rich clusters $([\mathrm{Fe} / \mathrm{H}]>-1.0)$ which appear to have too blue an HB morphology for their metallicity (B045, G1 and B158). In particular, G1 is a confirmed case of a metal-rich cluster with both red and blue HB stars (see Meylan et al. 2001).

This feature suggests a similarity with NGC 6441 and NGC 6388, the metal-rich Galactic GCs displaying a strong red HB clump as well as a significant blue HB tail, that it is currently interpreted as due to a large spread in He abundance (Caloi \& D'Antona 2007; Yoon et al. 2008). Also several bright GCs in M87 are interpreted as hosting a He abundance spread (see Rey et al. 2007, their Fig. 9, in particular). Therefore, similarly to what is believed to happen in some (likely most) Galactic GCs (Gratton et al. 2010, 2012, and references therein), also these M 31 GCs might host (multiple) populations of He-enriched stars, which evolve as Blue HB stars in spite of their high metallicity, as already proposed by, e.g. D'Antona et al. (2005). The occurrence of the same phenomenon in some massive and metalrich M 31 clusters has already been suggested with independent observational evidence by Rey et al. (2007); Colucci et al. (2009); Dalessandro et al. (2012). The pretty blue $(F U V-V)_{0}$ color of many Sample B clusters, possibly indicative of the presence of unseen BHB/EHB populations, has been already discussed in Sect. 2.2.1, above. We stress again that Sample B cluster is mainly composed by luminous/massive (18 on 25 have $M_{V} \leq-9.0$ ), a range of luminosity where only a few Galactic GCs (and very peculiar ones, like M54, $\omega$ Cen, NGC2419, etc.) are found.

It may be interesting to explore the available photometric and spectroscopic databases in search for further hints on the possible existence of multiple stellar populations in M $31 \mathrm{GCs}$. Such an analysis is in progress, and the results will be reported in a dedicated paper (Fusi Pecci et al., in prep.).

\section{Summary and discussion}

We performed the first thorough and homogeneous comparison of the HB morphology between M 31 and Milky Way globular clusters, based on HB star counts on cluster CMDs. We used a simplified version of the Mironov's index (SMI) that has been demonstrated to correlate very well with the popular HBR index. Strict requirements on the quality of the CMD forced us to limit the core of our analysis to a selected sample of 23 M 31 GCs, mostly located in the outer halo of M 31 (Sample A).

We find that eleven of these clusters lie on a significant different locus in the metallicity vs HB morphology plane with respect to their Galactic counterparts located at any distance from the MW center. Having an unusually red HB morphology for their metallicity, we refer to them as ARSA clusters (see Sect. 3). The possibility that such a difference arose from systematic errors in some of the involved parameters has been considered and dismissed as very unlikely.
The most straightforward interpretations of the observed difference are in terms of age or He abundance differences:

1. the ARSA clusters are, on average, $\approx 1$ Gyr younger than the MW Outer Halo GCs, and $\approx 2$ Gyr younger than the MW inner halo GCs; or

2. they have a different (internal) distribution of He abundances $(Y)$ than their Galactic counterparts, i.e. they should have a lower fraction of He-enriched stars (see Sect. 3).

Hypothesis 1 seems more natural, by analogy with the Milky Way (Dotter et al. 2011). In the Galaxy, slightly younger GCs are generally found to be associated with halo substructures and/or relics of disrupting dwarf galaxies (Marin-Franch et al. 2009). Also most ARSA clusters can be associated to similar substructures in the M 31 halo (Perina et al. 2009; Mackey et al. 2010), and hence younger ages would fit as a general characteristics of recently accreted clusters from dwarf satellites ${ }^{9}$. The fact that Sample A clusters are even younger than the MW OH clusters may be connected to the more complex and extended recent accretion activity that took place in Andromeda (w.r.t. the MW, Ibata et al. 2007). In the process of hierarchical assembly of the MW and M 31, the most massive (and most metal rich) sub-units were likely the first to merge at the bottom of the potential well, the strong compression shocks likely leading to a large amount of star (and cluster) formation on a short timescale. At later times less massive and more metal-poor fragments (dwarf galaxies) were accreted: these were able to form stars over longer timescales (Mateo 1998; Tolstoy et al. 2009). It can be conceived that also the epoch of GC formation was consequently postponed in these galaxies. In this context it is very interesting to note that, in the $[\mathrm{Fe} / \mathrm{H}]$ vs. HB morphology plane, dwarf galaxy satellites of the MW and M 31 lie virtually in the same locus as ARSA clusters (Yang \& Sarajedini 2012).

Perhaps the simplest way to envisage hypothesis 2 is to consider the possibility that the ARSA clusters do not host multiple populations, as most MW GCs do, or had a less pronounced early-enrichment history, hence do not present the sizable He abundance spread which acts as an internal second parameter, by pushing a fraction of cluster stars to the blue side of the HB. It has been suggested that clusters can host multiple populations (and consequently He enrichment) only above a certain mass threshold (a few $10^{4} M_{\odot}$, corresponding to $M_{V} \approx$ -5.5 according to Gratton et al. 2012, and references therein). Only one Sample A cluster is below this threshold, while $\approx 80 \%$ of the sample have $M_{V} \leq-7.0$ and $\approx 65 \%$ are brighter than the typical average of GC distributions $\left(M_{V}=-7.5\right.$, Brodie \& Strader 2006), hence they appear as massive as Galactic clusters where light element (and thus presumably $\mathrm{He}$ ) enrichment has been observed. Moreover, many ARSA clusters present bimodal HB morphology, possibly suggesting that some intrinsic difference among cluster stars is indeed there (but see Fusi Pecci et al. 1993, for serious warning on simplistic interpretations of complex morphologies). The formation environment may also have played a role (Bekki 2011), and the physical conditions that led to a later formation epoch may have also influenced the formation path of these clusters. Therefore, it is also possible that both age and $\mathrm{He}$ abundance have concurred in producing the observed difference in HB morphology between outer M 31 GCs and Milky Way clusters at any distance from the Galactic center. It is worth noting that the only Galactic GC in our sample

\footnotetext{
9 For example, it is interesting to note that the ARSA clusters MCGC8 and MCGC9 are both likely associated with the halo filamentary substructure called Eastern Arc (Stream D, in the nomenclature by Mackey et al. 2010).
} 
that lies on the same isochrone as the ARSA clusters, because it has a genuinely redder HB morphology than other clusters of the same metallicity (NGC 4590; see Sect. 3), is as old as them. Therefore, at least in this case, the difference in HB morphology must be due to some other parameter than age (see, e.g. Dalessandro et al. 2012).

Finally, the analysis of sample B clusters provides support to the conclusions by Rey et al. (2007) and Dalessandro et al. (2012) that a population of metal-rich GCs with sizable fractions of Blue $\mathrm{HB}$ stars is present in M 31, possibly including more extreme cases with respect to their Galactic counterparts (i.e. NGC 6388, NGC 6441), since they show larger SMI values. NGC 6388 and NGC 6441 have a strongly bimodal HB morphology that is difficult to explain without invoking a significant spread in He abundance among cluster stars (Caloi \& D' Antona 2007; Yoon et al. 2008). Also, many Sample B clusters have $(F U V-V)_{0}$ colors suggesting that the presence of extended blue HB tails may be more common in M 31 clusters than in the Milky Way. However, it must be noted that the majority of sample B clusters are very bright ( $72 \%$ brighter than $M_{V}=-9.0$ and only one cluster with $M_{V}>-8.0$ ). Carretta et al. (2010) found that more luminous/massive clusters appear to have wider distributions of light elements abundance: this should lead to wider distributions of $Y$ and, consequently, to more extended blue tails in the HB. Hence, the above result may simply reflect the case that very massive clusters, possibly with strong anti-correlation patterns and complex HB morphologies including extended blue tails (and blue hooks), like $\omega$ Cen or M 54, are much more numerous in the Andromeda galaxy than in the Milky Way ${ }^{10}$. Whatever the physical mechanism that led to such UV-enhanced HB morphology, its better knowledge in the M 31 GC environment could certainly add important clues to a better understanding of the early evolution of the whole cluster system around our nearby galaxy companion, in spite of all the observing problems associated with its large distance. Furthermore, on a larger galactic mass scale, this scenario could also shed light to the well recognized UV-upturn phenomenon, that in most cases seems to constrain the evolution of elliptical galaxies (Yi et al. 2011; Buzzoni \& Gonzalez-Lopezlira 2008; Buzzoni et al. 2012).

Acknowledgements. We acknowledge the financial support to this research by INAF through the PRIN-INAF 2009 grant CRA 1.06.12.10 (PI: R. Gratton) and by ASI through contracts COFIS ASI-INAF I/016/07/0 and ASI-INAF I/009/10/0. We are grateful to Marcio Catelan and Emanuele Dalessandro for useful suggestions and discussion. Emanuele Dalessandro kindly shared their data with us before publication.

\section{References}

Ajhar, E. A., Grillmair, C. J., Lauer, T. R., et al. 1996, AJ, 111, 1110 Barmby, P., Huchra, J. P., Brodie, J. P., et al. 2000, AJ, 119, 727 Barmby, P., Huchra, J. P., \& Brodie, J. P. 2001, AJ, 121, 1482 Bekki, K. 2011, MNRAS, 412, 2241

Brodie, J. P., \& Strader, J. 2006, ARA\&A, 44, 193

Brown, T. M., Ferguson, H. C., Smith, E., et al. 2004, ApJ, 613, L125 Buzzoni, A. 1995, ApJS, 98, 69

Buzzoni, A., \& Gonzalez-Lopezlira, R. A. 2008, ApJ, 686, 1007

Buzzoni, A., Bertone, E., Carraro, G., \& Buson, L. 2012, ApJ, 749, 35

Caldwell, N., Schiavon, R., Morrison, H., Rose, J. A., \& Harding, P. 2011, AJ, 141,61

Caloi, V., \& D'Antona, F. 2007, A\&A, 463, 949

\footnotetext{
10 This does not necessarily mean that the fraction of extended-bluetail clusters is higher in M 31 than in the MW, since M 31 host a much larger number of GCs than the MW ( $\gtrsim 450$ vs. $\approx 150$; Barmby et al. 2001; Galleti et al. 2006).
}

Catelan, M. 1993, A\&AS, 98, 547

Catelan, M. 2009, A\&SS, 320, 261

Catelan, M., \& de Freitas Pacheco, J. A. 1994, A\&A, 289, 394

Catelan, M., \& de Freitas Pacheco, J. A. 1995, A\&A, 297, 345

Carretta, E., Bragaglia, A., Gratton, R. G., et al. 2010, A\&A, 516, A55

Colucci, J. E., Bernstein, R. A., Cameron, S., McWilliam, A., \& Cohen, J. G. 2009, ApJ, 704, 385

Dalessandro, E., Schiavon, R. P., Rood, R. T., et al. 2012, AJ, in press [arXiv: 1208.5698]

D’Antona, F., Bellazzini, M., Caloi, V., et al. 2005, ApJ, 631, 868 Demarque, P., Zinn, R., Lee, Y.-W., \& Yi, S. 2000, AJ, 119, 1398

Dotter, A., Sarajedini, A., Anderson, J., et al. 2010, ApJ, 708, 698 Dotter, A., Sarajedini, A., \& Anderson, J. 2011, ApJ, 738, 74

Fan, Z., Ma, J., de Grijs, R., \& Zhou, X. 2008, MNRAS, 385, 1973

Federici, L., Cacciari, C., Bellazzini, M., et al. 2012, A\&A, 544, A155 (Paper I)

Ferraro, F. R., Messineo, M., Fusi Pecci, F., et al. 1999, AJ, 118, 1738

Freeman, K. C., \& Bland-Hawthorn, J. 2002, ARA\&A, 40, 487

Fusi Pecci, F., \& Bellazzini, M. 1997, in The Third Conference on Faint Blue Stars, eds. A. G. D. Philip, J. Liebert, \& R. A. Saffer (Schenectady: Davis), 255

Fusi Pecci, F., Ferraro, F. R., Bellazzini, M., et al. 1993, AJ, 105, 1145

Fusi Pecci, F., Buonanno, R., Cacciari, C., et al. 1996, AJ, 112, 1461

Gallart, C., Zoccali, M., \& Aparicio, A. 2005, ARA\&A, 43, 387

Galleti, S., Federici, L., Bellazzini, M., Fusi Pecci, F., \& Macrina, S. 2004, A\&A, 416, 917 [RBCV4.0]

Galleti, S., Federici, L., Bellazzini, M., Buzzoni, A., \& Fusi Pecci, F. 2006, ApJ 650, L107

Galleti, S., Bellazzini, M., Buzzoni, A., Federici, L., \& Fusi Pecci, F. 2009, A\&A, 508, 1285

Gratton, R. G., Carretta, E., Bragaglia, A., Lucatello, S., \& D’Orazi, V. 2010, A\&A, 517, A81

Gratton, R., Carretta, E., \& Bragaglia, A. 2012, A\&ARv, 20, 50

Jasniewicz, G., \& Parthasarathy, M. 2009, in Globular Clusters - Guides to Galaxies, ESO Astrophysics Symposia (Berlin, Heidelberg: Springer), 35

Harris, W. E. 1996, AJ, 112, 1487

Holland, S., Fahlman, G. G., \& Richer, H. B. 1997, AJ, 114, 1488

Huxor, A. P., Tanvir, N. R., Irwin, M., et al. 2004, ASP Conf. Ser., 327, 118

Huxor, A. P., Tanvir, N. R., Irwin, M. J., et al. 2005, MNRAS, 360, 1007

Kang, Y., Rey, S.-C., Bianchi, L., et al. 2012, ApJS, 199, 37

Ibata, R. A., Martin, N. F., Irwin, M., et al. 2007, ApJ, 671, 1591

Lee, Y.-W., Demarque, P., \& Zinn, R. 1994, ApJ, 423, 248

Mackey, A. D., Huxor, A. P., Ferguson, A. M. N., et al. 2006, ApJ, 653, L105 [M06]

Mackey, A. D., Huxor, A. P., Ferguson, A. M. N., et al. 2007, ApJ, 655, L85 [M07]

Mackey, A. D., Huxor, A. P., Ferguson, A. M. N., et al. 2010, ApJ, 717, L16

Marin-Franch, A., Aparicio, A., Piotto, G., et al. 2009, ApJ, 694, 1498

Mateo, M. 1998, ARA\&A, 36, 435

Meylan, G., Sarajedini, A., Jablonka, P., et al. 2001, AJ, 122, 830

McConnachie, A. W., Irwin, M. J., Ferguson, A. M. N., et al. 2005, MNRAS, 356,979

Mironov, A. V. 1972, SvA, 16, 105

Perina, S., Federici, L., Bellazzini, M., et al. 2009, A\&A, 507, 1375

Perina, S., Galleti, S., Fusi Pecci, F., et al. 2011, A\&A, 531, A155

Piotto, G., King, I. R., Djorgovski, S. G., et al. 2002, A\&A, 391, 945

Preston, G. W., Shectman, S. A., \& Beers, T. C. 1991, ApJ, 375, 121

Recío-Blanco, A., Aparicio, A., Piotto, G., de Angeli, F., \& Djorgovski, S. G. 2006, A\&A, 452, 875

Renzini, A., \& Fusi Pecci, F. 1988, ARA\&A, 26, 199

Rey, S.-C., Yoon, S. J., Lee, Y.-W., Chaboyer, B., \& Sarajedini, A. 2001, AJ, 122,3219

Rey, S.-C., Rich, R. M., Sohn, S. T., et al. 2007, ApJS, 173, 2007

Rich, R. M., Corsi, C. E., Cacciari, C., et al. 2005, AJ, 129, 2670

Rood, R. T. 1973, ApJ, 184, 815

Sandage, A., \& Wildey, R. 1067, ApJ, 150, 469

Schiavon, R., Dalessandro, E., Sohn, S. T., et al. 2012, AJ, 143, 121

Searle, L., \& Zinn, R. 1978, ApJ, 225, 790

Tolstoy, E., Hill, V., \& Tosi, M. 2009, ARA\&A, 47, 371

van den Bergh, S. 1994, AJ, 108, 2145

Yang, S.-C., \& Sarajedini, A. 2012, MNRAS, 419, 1362

Yoon, S.-J., Joo, S.-J., Ree, C. H., et al. 2008, ApJ, 677, 1080

Yi, S. K., \& Yoon, S.-J. 2004, Ap\&SS, 291, 205

Yi, S. K., Demarque, P., Kim, Y.-C., et al. 2001, ApJS, 136, 417

Yi, S. K., Lee, J., Sheen, Y.-K., et al. 2011, ApJS, 195, 22

Zinn, R. 1980, ApJ, 241, 602

Zinn, R., \& West, M. J. 1984, ApJS, 55, 45

Zinn, R. 1993, in The Globular Cluster-Galaxy Connection, eds. G. H. Smith, \& J. P. Brodie (San Francisco: ASP), ASPCS, 48, 38 